\title{
Digestibilidade in vivo dos nutrientes de cultivares de amoreira (Morus alba L.) em caprinos
}

\author{
Digestibility in vivo of the nutrients from mulberry (Morus alba L.) cultivates in goats
}

\author{
Cláudia Josefina Dorigan ${ }^{1}$ Kleber Tomás De Resende ${ }^{2}$ Roberta Basaglia ${ }^{3}$ Atushi Sugohara ${ }^{2}$ \\ Roque Takahashi $^{2}$ Roberto Germano Costa $^{4}$ Vânia Rodrigues Vasconcelos ${ }^{5}$
}

\section{RESUMO}

\begin{abstract}
Este trabalho foi conduzido para avaliar a digestibilidade "in vivo" dos nutrientes dos cultivares de amoreira FM 86 e FM SM nas idades de crescimento de 45 e 90 dias. Foram utilizados 8 caprinos machos, da raça Saanen, com 6 meses de idade e peso vivo médio de 26kg. O delineamento experimental adotado foi o inteiramente casualizado em esquema fatorial $2 \times 2$ (2 cultivares de amoreira e 2 idades de crescimento). O coeficiente de digestibilidade da FDN do cultivar FM 86 (74,82\%) superou o cultivar FM SM $(69,36 \%)$, não ocorrendo diferença significativa entre as idades de crescimento. Ocorreu interação significativa entre cultivar e idade de crescimento para o Coeficiente de Digestibilidade da FDA, e o cultivar FM 86 na idade de 45 dias (75,09\%) superou a de 90 dias (68,82\%). Para os parâmetros NDT e oeficientes de digestibilidade da energia, MS e PB, verificou-se superioridade da idade de corte de 45 dias, sem diferença entre os cultivares. Concluiuse que os cultivares FM 86 e FM SM apresentaram excelente valor energético e altos coeficientes de digestibilidade da $M S$, da PB e dos constituintes da parede celular, indicando um elevado potencial da amoreira como forrageira para caprinos.
\end{abstract}

Palavras-chave: nutrição, avaliação de alimentos, volumoso.

\section{ABSTRACT}

This work was carried out for evaluating in vivo digestibility of nutrients from FM 86 and FM SM cultivars of mulberry at two growth ages, 45 and 90 days. Eight Saanen male goats being six month-old and $26 \mathrm{~kg}$ of live weight were used. Experimental design was completely randomized factorial $2 \times 2$ (two mulberry cultivars and two growth ages). The NDF digestibility coefficient of FM 86 cultivar (74.82\%) was higher than the FM SM (69.36\%) and there was not statiscal difference between their growth ages. In relation to $A D F$ digestibility coefficient, there was significant interaction between cultivars and growth ages. FM 86 cultivar with 45 day-old showed ADF digestibility coefficient higher (75.09\%) than that with 90 day-old (68.82\%). Considering NDT and energy, DM and CP digestibility coefficients, it was observed the superiority of plants with 45 day-old, independently of the cultivar. It was concluded that FM 86 and FM SM cultivars showed great energetic values and high DM, CP and cellular wall constituents digestibility coefficients which indicated the elevated power of mulberry for using as forage in goat nutrition.

Key words: nutrition, feed evaluation, roughage

\section{INTRODUÇÃO}

Tanto em regiões de agricultura intensiva e densa população humana como em outras menos evoluídas e com menor população, a cabra tem uma importante função econômica como elaboradora de bons produtos destinados ao consumo humano, tais como carne e leite (JARDIM, 1992), fornecendo, ainda, matéria prima para confecção de vestuário. Mesmo assim, alguns aspectos da nutrição e alimentação dos caprinos são pouco conhecidos, visto que existem

${ }^{1}$ Zootecnista Doutor em Produção Animal, Praça Dulio Poli 154, 14870-000, Jaboticabal SP. E-mail: cdorigan@ netsite.com.br. Autor para correspondência.

${ }^{2}$ Professor Departamento de Zootecnia da Faculdade de Ciências Agrárias e Veterinárias, UNESP, Campus de Jaboticabal.

${ }^{3}$ Zootecnista.

${ }^{4}$ Professor, Universidade Federal da Paraíba, PB.

${ }^{5}$ Pesquisador, CNPC/EMBRAPA. 
trabalhos insuficientes que objetivam essa particularidade, às vezes fazendo-se necessário extrapolar os dados de trabalhos encontrados para outras espécies animais, como bovinos e ovinos.

Os caprinos são pequenos ruminantes que apresentam elevada flexibilidade alimentar. São considerados selecionadores intermediários que consomem preferencialmente dicotiledôneas herbáceas, brotos e folhas de árvores e arbustos (VAN SOEST, 1982). A base de sua alimentação é constituída por plantas forrageiras, que apresentam a produção comprometida nas épocas de escassez de chuvas, o que faz necessária a avaliação de espécies vegetais como alternativas potencialmente produtivas nessas épocas críticas.

Dessa forma, a amoreira que é uma planta rústica e de raízes profundas, que produz quantidades satisfatórias de matéria seca, mesmo na época seca, surge como uma alternativa alimentar interessante. É pouco exigente quanto a tipos de solo, além de ser considerada uma excelente fonte protéica (TAKAHASHI,1988). CARNAZ (1992) obteve produção média de matéria seca de folhas de $6141 \mathrm{~kg}$ ha $^{-1}$ e $4973 \mathrm{~kg} \mathrm{ha}^{-1}$, respectivamente para os híbridos FM 86 e FM Shima Miúra, plantados em espaçamento de $3 \mathrm{~m}$ entre linhas e $0,6 \mathrm{~m}$ entre plantas.

Além das características relacionadas à produção, outro fator importante, que deve ser considerado para a escolha de uma planta forrageira, é a digestibilidade de seus nutrientes, ou seja, quanto do que foi ingerido que poderá ser aproveitado pelo organismo animal. Sendo assim, TAKAHASHI et al. (1994) determinaram a digestibilidade "in vitro" do cultivar de amoreira Yamada em quatro idades de crescimento (45, 60, 75 e 90 dias). A digestibilidade da matéria seca da fração caule foi maior na idade de 45 dias (52,8\%); enquanto a digestibilidade da fração folha foi superior na idade de 60 dias $(83,4 \%)$. Quanto à digestibilidade da proteína bruta (PB), na fração caule, não ocorreu diferença entre as idades, obtendo-se valor médio de $93,4 \%$. O corte aos 90 dias apresentou a mais baixa digestibilidade da PB para a fração folha (86\%), sendo que a média dos demais cortes foi $88 \%$. Outro trabalho em que se determinou a digestibilidade "in vitro" foi conduzido por RESENDE et al. (1994a), no qual se avaliou caule e folhas nas mesmas épocas de corte nos cultivares FM Shima Miúra e FM 86, encontrando-se valores médios de $48 \%$ (fração caule) e 70,0\% (fração folha) para o coeficiente de digestibilidade da matéria seca, sendo que essa digestibilidade diminuiu, na fração caule, com o aumento da idade da planta, fato que não aconteceu com a digestibilidade da fração folha, a qual foi semelhante nas idades de 45 e 90 dias, com valor médio de 70,0\%. O coeficiente de digestibilidade da proteína bruta apresentou valor médio de $65,9 \%$, para a fração folha, ocorrendo também variação nesse coeficiente com o avançar da idade da planta. O maior valor $(75,6 \%)$, foi obtido no corte aos 60 dias.

Uma vez que o aproveitamento do alimento volumoso é realizado quase que totalmente pela população microbiana do rúmen, é importante considerarmos resultados de trabalhos que objetivam esta particularidade. Dessa forma, a degradação ruminal, em caprinos, de dois cultivares de amoreira (FM Shima Miúra e FM 86), com 45 e 90 dias de crescimento, por meio da digestibilidade "in situ", foi estudada por VASCONCELOS (1994). A degradação da matéria seca e da proteína bruta não diferiu significativacamente entre os cultivares. Ocorreram, no entanto, diferenças significativas para esses parâmetros, em relação à idade de crescimento, sendo que os resultados obtidos para a idade de 45 dias foram superiores aos encontrados para a idade de 90 dias. Com relação à degradação da fibra em detergente neutro, foi observada diferença significativa entre cultivares, com superioridade do FM 86 (65,7\%).

Diante do exposto, o presente trabalho teve como objetivo determinar a digestibilidade "in vivo" dos nutrientes dos híbridos de amoreira, FM 86 e FM Shima Miúra (FM SM), pelos caprinos, nas idades de crescimento de 45 e 90 dias.

\section{MATERIAL E MÉTODOS}

O trabalho foi desenvolvido na Faculdade de Ciências Agrárias e Veterinárias de Jaboticabal UNESP, utilizando-se 8 caprinos machos da raça Saanen, castrados, com aproximadamente 6 meses de idade e peso médio de $26 \mathrm{~kg}$, nos meses de janeiro e fevereiro.

Realizou-se um ensaio de digestibilidade in vivo, constituído por dois períodos de coleta com duração de cinco dias cada um, para avaliar os nutrientes dos cultivares de amoreira (FM 86 e FM SM), em duas idades de crescimento (45 e 90 dias). Este alimento foi fornecido aos caprinos exclusivamente na forma de feno, formado pela fração folha da planta constituída pelo limbo, pecíolo e região apical do caule, que foram destacadas com uma leve tração manual. Dessa forma, os tratamentos foram os seguintes; T1: FM 86 - 45 dias, T2: FM 86 90 dias, T3: FM SM - 45 dias e T4: FM SM - 90 dias. Antes do início das coletas, os caprinos permaneceram por 21 dias em período de adaptação às dietas e instalações.

Antes de cada período, os tratamentos foram sorteados aleatoriamente entre os oito animais, 
resultando em duas repetições de tratamento em cada período de coleta. O consumo voluntário de feno foi estimado no decorrer do período de adaptação em $51,2 \mathrm{~g} / \mathrm{kg}^{0,75}(2,3 \% \mathrm{PV})$, por meio do controle diário de ingestão de alimentos.

Durante o período experimental, os animais permaneceram alojados em gaiolas para estudo de metabolismo. As coletas de fezes e urina foram realizadas duas vezes ao dia. Amostras representativas de fezes $(20 \%)$ foram secas em estufas com circulação forçada de ar e temperatura de $60^{\circ} \mathrm{C}$, e as amostras de urina armazenadas em um freeser a $-12^{\circ} \mathrm{C}$.

As amostras de alimento, fezes e urina foram analisadas no Laboratório de Nutrição Animal da FCAVUNESP, onde foram determinados os teores de matéria seca (MS), proteína bruta (PB), fibra em detergente neutro (FDN), fibra em detergente ácido (FDA), celulose (Cel), hemicelulose (Hcel), energia bruta (EB), extrato etéreo (EE), extrativos não nitrogenados (ENN); de acordo com a metodologia descrita em SILVA (1981).

Avaliaram-se os seguintes parâmetros: coeficientes de digestibilidade da matéria seca (CDMS), coeficientes de digestibilidade da proteína bruta (CDPB), nutrientes digestíveis totais (NDT), coeficientes de digestibilidade da energia (CDE), coeficientes de digestibilidade da fibra em detergente ácido (CDFDA), coeficientes de digestibilidade da fibra em detergente neutro (CDFDN), coeficientes de digestibilidade da hemicelulose (CDHcel), coeficientes de digestibilidade da celulose (CDCEL).

Utilizou-se inicialmente o delineamento experimental inteiramente casualizado em esquema fatorial 2 x 2 (dois cultivares de amoreira e duas idades de crescimento), com dois períodos de coleta e duas repetições dentro de período. O modelo matemático utilizado foi:

Yijk $=\mu+\mathrm{Ai}+\mathrm{Cj}+\mathrm{Acij}+$ eijk, em que:

Yijk = observações referentes ao híbrido de amoreira $\mathrm{i}$, com idade de corte $\mathrm{j}$;

$\mu=$ efeito da média;

$\mathrm{Ai}=$ efeito do híbrido de amoreira;

$\mathrm{Cj}=$ efeito da idade de corte $\mathrm{j}$;

Acih = efeito da interação A x C;

eijk = erro aleatório.

Os resultados obtidos foram analisados por meio do SAS (1989), e quando ocorreu interação entre os fatores, estes foram comparados por meio do teste de Tukey $(5 \%)$.

\section{RESULTADOS}

A tabela 1 apresenta os valores referentes à composição químico-bromatológica dos cultivares de amoreira FM 86 e FM SM nas idades de crescimento de 45 e 90 dias. Foi verificado que as percentagens dos constituintes da parede celular apresentaram pequenas variações nos dois cultivares, tanto na idade de 45 quanto na de 90 dias. A lignina, que pode influenciar negativamente a utilização dos demais constituintes fibrosos, teve sua participação aumentada em 1,3\% no cultivar FM 86 e 1,93\% no cultivar FM SM. Os teores de proteína bruta também decresceram em pequena proporção: para o cultivar FM 86 de $25,33 \%$ para $21,09 \%$ e para o cultivar FM SM de $24,84 \%$ para $22,60 \%$.

Com o avançar da idade da planta, era esperado que ocorresse redução nos coeficientes de digestibilidade dos componentes da parede celular, pois o aumento no teor de carboidratos estruturais influenciam negativamente na eficiência de utilização dos alimentos. Entretanto, a variação obtida no presente trabalho foi reduzida, o que pode ser explicado pela pequena variação na composição bromatológica dos cultivares de amoreira (Tabela 1).

Comparando-se os resultados obtidos para a FDN (Tabela 1) com aqueles obtidos por RESENDE et al. (1994b), que trabalharam com o cultivar Yamada em 4 idades de crescimento, em dois ensaios, observase que esses são menores, ou seja: no primeiro ensaio: observaram-se variações nas percentagens de FDN de 23,$97 ; 23,85$ e $25,64 \%$ para 60,75 e 90 dias de crescimento, e no segundo ensaio 25,$30 ; 24,68 ; 21,59 \mathrm{e}$ 29,94\% para 45, 60, 75 e 90 dias de crescimento, também para os percentuais de FDN. SUGOHARA (1994) trabalhando, também, com o cultivar Yamada, observou nas idades de crescimento de 45 e 90 dias, teores de FDN de 34,47 e 33,34\%; de FDA: 22,25 e 22,17\%; de lignina 9,02 e $8,87 \%$; de celulose: 5,92 e $9,53 \%$ de e EE: 3,75 e $5,20 \%$, respectivamente, concluindo que estes resultados evidenciaram que as idades estudadas pouco afetaram o valor nutritivo da amoreira, o que implica facilidade de manejo da cultura, podendo assim, ser conservada no campo com ótima composição em nutrientes.

Os valores encontrados para CDFDN (\%), CDFDA (\%), CDCEL (\%),CDHCEL (\%), encontram-se na tabela 2. O CDFDN (\%) do cultivar FM 86 foi maior $(\mathrm{P}<0,05)$ do que o do cultivar FM SM. Entre as idades de crescimento, no entanto, não ocorreu diferença significativa para este coeficiente. O parâmetro CDHCEL foi semelhante entre os cultivares e as idades de crescimento $(P>0,05)$. Ocorreu interação significativa $(\mathrm{P}>0,05)$ entre os fatores cultivar e idade de crescimento para os CDFDA e CDCEL, sendo que o cultivar FM 86 com 90 dias de crescimento 
Tabela 1 - Composição bromatológica do feno dos cultivares de amoreira FM 86 e FM Shima-Miúra (FM SM) nas idades de crescimento de 45 e 90 dias

\begin{tabular}{|c|c|c|c|c|}
\hline \multirow{2}{*}{ Constituintes } & \multicolumn{2}{|c|}{ FM 86} & \multicolumn{2}{|c|}{ FM SM } \\
\hline & 45 & 90 & 45 & 90 \\
\hline Matéria seca (\%) & 82,65 & 82,05 & 83,49 & 80,98 \\
\hline Fibra bruta $(\%)$ & 10,73 & 10,21 & 9,21 & 11,26 \\
\hline Fibra em detergente ácido (\%) & 19,29 & 18,97 & 17,23 & 18,62 \\
\hline Fibra em detergente neutro (\%) & 24,94 & 25,11 & 21,85 & 22,19 \\
\hline Celulose (\%) & 13,56 & 12,40 & 10,96 & 11,19 \\
\hline Hemicelulose $(\%)$ & 5,65 & 6,14 & 4,62 & 3,57 \\
\hline Lignina $(\%)$ & 4,58 & 5,88 & 4,29 & 6,22 \\
\hline Proteína Bruta (\%) & 25,33 & 21,09 & 24,84 & 22,60 \\
\hline Extrato Etéreo (\%) & 4,72 & 5,44 & 4,98 & 5,01 \\
\hline Extrativos não nitrogenados (\%) & 49,17 & 53,02 & 51,34 & 51,12 \\
\hline Energia bruta (Kcal/kg) & 4038,76 & 4046,36 & 4100,88 & 4147,05 \\
\hline Energia digestível (Kcal/kg) & 3114,29 & 2705,40 & 3080,17 & 2805,48 \\
\hline Energia metabolizável (Kcal/kg) & 2261,30 & 1276,14 & 2315,77 & 2059,01 \\
\hline Cinza $(\%)$ & 10,05 & 10,24 & 9,62 & 10,01 \\
\hline
\end{tabular}

apresentou valores mais baixos para estas duas variáveis. Os resultados obtidos para o CDFDN, concordam com os observados por VASCONCELOS et al. (1994) quando compararam esses mesmos cultivares por meio de um ensaio de digestibilidade "in situ" e também obtiveram superioridade para o FM86, com coeficiente médio de 65,70\%.

$\mathrm{Na}$ tabela 3, encontram-se os dados referentes ao NDT (\%) e coeficientes de digestibilidade da MS (\%), da PB (\%) e da energia (\%), dos híbridos de amoreira nas diferentes idades de crescimento. Para o CDMS (\%), CDE (\%) e NDT (\%) não ocorreram diferenças entre os híbridos $(\mathrm{P}>0,05)$, mas sim entre as idades de corte $(\mathrm{P}<0,05)$. Para o CDPB $(\%)$, ocorreu interação entre os dois fatores estudados $(\mathrm{P}<0,05)$. Para ambos os cultivares, o CDPB foi superior para a idade de corte de 45 dias.

Os resultados obtidos para o coeficiente de digestibilidade da matéria seca (Tabela 3), para cultivar, estão de acordo com RESENDE et al. (1994a) que também não observaram diferença significativa na digestibilidade "in vitro" da matéria seca das folhas dos cultivares Korin, Calabresa, FM 86, FM Shima Miura e Miura. Por outro lado, estes mesmos autores,

Tabela 2 - Coeficientes de digestibilidade da fibra em detergente neutro (CDFDN), da fibra em detergente ácido (CDFDA), da celulose (CDCEL), da hemicelulose (CDHCEL) dos cultivares de amoreira FM 86 e FM SM, nas idades de crescimento de 45 e 90 dias

\begin{tabular}{|c|c|c|c|c|c|}
\hline \multirow{2}{*}{ Parâmetro } & \multirow{2}{*}{ Cultivar } & \multicolumn{2}{|c|}{ Idades de Crescimento } & \multirow{2}{*}{ Média } & \multirow{2}{*}{$\mathrm{CV}(\%)$} \\
\hline & & 45 & 90 & & \\
\hline \multirow{3}{*}{ CDFDN (\%) } & FM 86 & 77,36 & 72,29 & $74,82 \mathrm{~A}$ & \multirow{3}{*}{4,42} \\
\hline & FM SM & 68,94 & 69,79 & $69,36 \mathrm{~B}$ & \\
\hline & MÉDIA & $73,15 \mathrm{~A}$ & $71,04 \mathrm{~A}$ & - & \\
\hline \multirow{3}{*}{ CDFDA (\%) } & FM 86 & 75,09 a & $68,82 \mathrm{~b}$ & 71,95 & \multirow{3}{*}{3,18} \\
\hline & FM SM & $68,35 \mathrm{a}$ & $68,30 \mathrm{a}$ & 68,32 & \\
\hline & MÉDIA & 71,72 & 68,56 & - & \\
\hline \multirow{3}{*}{ CDCEL $(\%)$} & FM 86 & 81,93 a & $77,34 \mathrm{~b}$ & 79,64 & \multirow{3}{*}{3,27} \\
\hline & FM SM & 72,08 a & $75,21 \mathrm{a}$ & 73,65 & \\
\hline & MÉDIA & 77,01 & 76,28 & - & \\
\hline \multirow{3}{*}{ CDHCEL (\%) } & FM 86 & 85,09 & 83,03 & $84,06 \mathrm{~A}$ & \multirow{3}{*}{19,02} \\
\hline & FM SM & 71,14 & 77,59 & $74,35 \mathrm{~A}$ & \\
\hline & MÉDIA & $78,11 \mathrm{~A}$ & $80,30 \mathrm{~A}$ & - & \\
\hline
\end{tabular}

Letras iguais, nas linhas e colunas, para cada constituintes, não diferem entre si pelo teste $\mathrm{F}(\mathrm{P}>0,05)$. 
Tabela 3 - Coeficientes de digestibilidade da matéria seca (CDMS), da proteína bruta (CDPB), da energia (CDE) e nutrientes digestíveis totais (NDT), dos cultivares de amoreira FM 86 e FM SM nas idades de crescimento de 45 e 90 dias

\begin{tabular}{|c|c|c|c|c|c|}
\hline \multirow{2}{*}{ Parâmetro } & \multirow{2}{*}{ Cultivar } & \multicolumn{2}{|c|}{ Idades de Crescimento } & \multirow{2}{*}{ Média } & \multirow{2}{*}{$\mathrm{CV}(\%)$} \\
\hline & & 45 & 90 & & \\
\hline \multirow{3}{*}{ CDMS (\%) } & FM 86 & 78,22 & 71,38 & $74,80 \mathrm{~A}$ & \multirow{3}{*}{2,28} \\
\hline & FM SM & 76,80 & 70,83 & $73,82 \mathrm{~A}$ & \\
\hline & MÉDIA & $77,51 \mathrm{~A}$ & $71,10 \mathrm{~B}$ & - & \\
\hline \multirow{3}{*}{ CDPB (\%) } & FM 86 & $80,80 \mathrm{a}$ & $71,54 \mathrm{~b}$ & 76,67 & \multirow{3}{*}{3,06} \\
\hline & FM SM & 80,47 a & $75,60 \mathrm{~b}$ & 78,04 & \\
\hline & MÉDIA & 81,14 & 73,57 & - & \\
\hline \multirow{3}{*}{$\operatorname{CDE}(\%)$} & FM 86 & 77,11 & 66,86 & $71,98 \mathrm{~A}$ & \multirow{3}{*}{3,41} \\
\hline & FM SM & 75,11 & 67,65 & $71,30 \mathrm{~A}$ & \\
\hline & MÉDIA & $76,11 \mathrm{~A}$ & $67,26 \mathrm{~B}$ & - & \\
\hline \multirow{3}{*}{ NDT $(\%)$} & FM 86 & 76,88 & 64,69 & $70,79 \mathrm{~A}$ & \multirow{3}{*}{2,59} \\
\hline & FM SM & 73,93 & 65,65 & $69,79 \mathrm{~A}$ & \\
\hline & MÉDIA & $75,41 \mathrm{~A}$ & $65,17 \mathrm{~B}$ & - & \\
\hline
\end{tabular}

Letras iguais, nas linhas e colunas, para cada constituintes, não diferem entre si pelo teste $\mathrm{F}(\mathrm{P}>0,05)$.

não verificaram diferença significativa desses cultivares para as idades de crescimento de 45, 60, 75 e 90 dias após poda de uniformização, com os respectivos coeficientes: $66,91,68,70,66,60 \mathrm{e}$ $68,16 \%$.

O comportamento dos dados para idade de crescimento e coeficientes de digestibilidade da PB (Tabela 3) estão de acordo com TAKAHASHI et al. (1994), que estudando a digestibilidade "in vitro" da matéria seca do cultivar Yamada em diferentes idades de crescimento $(45,60,75$ e 90 dias, após poda de uniformização), observaram diferenças significativa entre eles $(82,65,83,27,79,95$ e $78,90 \%$, respectivamente). Para os coeficientes de digestibilidade da PB encontraram diferenças significativas entre várias idades de crescimento (45, 60, 75 e 90 dias, após poda de uniformização), com os respectivos valores: $58,27,58,76,51,96$ e $56,30 \%$.

Comparando a digestibilidade da proteína bruta da amoreira com a de leguminosas, verifica-se que os valores encontrados neste trabalho foram superiores aos citados por GOMIDE e QUEIROZ (1993) para leucena em ovinos, que variaram entre $43,2 \%$ a 57,6\%; e semelhantes aos citados por GOHL (1982) para feno de alfafa $(74,1 \%)$ e soja perene $(71,2 \%)$. Os valores obtidos para o NDT $(\%)$ podem ser considerados altos, uma vez que fenos de outras forrageiras como a alfafa, o guandu e a soja perene, apresentaram teores de NDT de 58,2, 57,6 e 52,7\%, respectivamente (PUPO, 1986).

\section{CONCLUSÕES}

De acordo com os resultados obtidos, conclui-se que:

Em função dos resultados de composição bromatológica e de digestibilidade dos nutrientes, os híbridos de amoreira estudados podem ser utilizados satisfatoriamente como alimento volumoso para caprinos.

\section{REFERÊNCIAS BIBLIOGRÁFICAS}

CARNAZ, L.F.F. Determinação da produção e perda de água de 6 cultivares de amoreira (Morus alba L). 1992. 42f. Monografia (Graduação em Zootecnia) - Faculdade de Ciências Agrárias e Veterinárias, UNESP.

GOHL, B. Piensos tropicales. Roma : Organizacion de las Naciones Unidas para la Agricultura y la Alimentación. 1982. p.169-183. (Coleción FAO: Producción y Sanidad Animal, 12.

GOMIDE, J.A.; QUEIROS, D.S. Valor nutritivo de leguminosas arboreas e arbustivas. IN: SIMPÓSIO SOBRE USOS MÚLTIPLOS DE LEGUMINOSAS ARBUSTIVAS E ARBÓREAS, 1., 1993, Nova Odessa. Anais... Nova Odessa: Instituto de Zootecnia, 1993. p.31-62.

JARDIM, W.R. Criação de caprinos. 4.ed. São Paulo: Melhoramentos, 1992. 306p.

PUPO, N.I.H. Manual de pastagens e forrageiras: Formação - conservação - utilização. Campinas : Instituto Campineiro de Ensino Agrícola, 1991. 343p.

REIS, R.A.; RODRIGUES, L.R.A. Valor nutritivo de plantas forrageiras. Jaboticabal : Fundação para o ensino e pesquisa, 1993 . 26p.

Ciência Rural, v.34, n.2, mar-abr, 2004. 
RESENDE, K.T. et al. Produção e digestibilidade de cultivares de amoreira (Morus alba L.). In : REUNIÃO ANUAL DA SBZ, 33., 1994, Maringá. Anais... Maringá, MG, 1994. p.526, $1994 b$.

RESENDE, K.T. et al. Composição bromatológica de cultivares de amoreira (Morus alba L.). In: REUNIÃO ANUAL DA SBZ, 33., 1994, Maringá. Anais... Maringá, MG, 1994a. p.532, 1994.

SILVA, D.J. Análise de alimentos. Viçosa : UFV, 1981. $166 \mathrm{p}$.

SUGOHARA, A. Composição bromatológica da amoreira (Morus alba L.) cultivar Yamada, em diferentes idades de crescimento. In: REUNIÃO ANUAL DA SBZ, 33., 1994, Maringá. Anais... Maringá, MG, 1994. p.532, 1994. Anais... Maringá, MG, 1994. p.525.
TAKAHASHI, R. Sericicultura. Jaboticabal : FCAV/UNESP, 1988. 135p. (Apostila).

TAKAHASHI, R. et al. Produção e digestibilidade da amoreira (Morus alba L.), cultivar Yamada, em diferentes idades de crescimento. In: REUNIÃO ANUAL DA SBZ, 33., 1994, Maringá. Anais... Maringá, MG, 1994. p.524.

VAN SOEST, P.J. Nutritional ecology of the ruminant. Oregon : O \& B Books, 1982. 374p.

VASCONCELOS, V.R. et al. Características de degradação ruminal da matéria seca e proteína bruta da amoreira (Morus alba L.) por caprinos. In: REUNIÃO ANUAL DA SBZ, 33., 1994, Maringá. Anais... Maringá, MG, 1994. p. 541 . 